\title{
Molecular classification of hepatocellular adenoma: A single-center experience
}

\author{
Xue-Yin Shen ${ }^{1}$, Xu-Guang $\mathrm{Hu}^{2}$, Young-Bae $\mathrm{Kim}^{3}$, Mi-Na Kim${ }^{1}$, Sung-Yeon Hong ${ }^{1}$, \\ Bong-Wan $\mathrm{Kim}^{1}$, and Hee-Jung Wang ${ }^{1}$
}

\begin{abstract}
${ }^{1}$ Division of Hepatobiliary Surgery and Liver Transplantation, Department of Surgery, Ajou University School of Medicine, Suwon, ${ }^{2}$ Department of Hepatobiliary Surgery, Jiangxi Cancer Center, Nanchang, China, ${ }^{3}$ Department of Pathology, Ajou University School of Medicine, Suwon, Korea
\end{abstract}

\begin{abstract}
Backgrounds/Aims: Hepatocellular adenoma $(\mathrm{HCA})$ is a rare benign tumor that has a risk of malignant transformation into hepatocellular carcinoma (HCC) and bleeding. The aim of this study was to analyze the characteristics of HCA by performing molecular classification. Methods: We retrospectively collected data from nine patients who were diagnosed with HCA from 1995 to 2016 . The patients underwent liver surgery due to the existence of clinical symptoms. Immunohistochemical (IHC) staining was performed to classify the subgroups of HCA. Results: Four patients with both $\beta$-catenin and inflammation were classified as $\beta$-IHCA. Two patients were defined as $\beta$-HCA. Two patients were classified as HHCA. Only one patient was defined as IHCA. None of the patients had unclassified HCA. Seven of nine patients had a malignant transformation. By comparing the characteristics of HCA between two groups, we found the mean tumor size in the malignant transformation group was greater than the non-malignant transformation group. Conclusions: Taken together, the mean tumor size and activation of catenin $\beta 1$ mutation status might be the risk factors for the malignant transformation of HCA into HCC. Moreover, IHCA without the catenin $\beta 1$ mutation could also have a possibility of malignant transformation into HCC. (Ann Hepatobiliary Pancreat Surg 2019;23:109-114)
\end{abstract}

Key Words: Adenoma; Liver Cell; Molecular pathology; Subtype; Carcinoma; Hepatocellular

\section{INTRODUCTION}

Hepatocellular adenoma (HCA) is a rare benign tumor that occurs frequently in women of reproductive age with a history of oral contraceptives (OCs) use. ${ }^{1}$ As a result of cultural differences, OCs are more commonly used in Western countries, leading to a higher incidence of HCA compared with that in Eastern countries. ${ }^{2-4}$ However, exposure to androgens or underlying metabolic syndrome including glycogen storage disease (GSD) type la and Fanconi anemia, which can also affect men, promotes HCA occurrence. ${ }^{5-7}$ Although benign, HCA can be complicated by rupture and hemorrhage and require surgical intervention. ${ }^{8}$ Hepatocellular carcinoma (HCC) may arise from HCA, which is known as malignant transformation. Malignant transformation is a rare event, with a reported incidence of $4.7-10.6 \%$ in patients with $\mathrm{HCA} .^{9-12}$

Recent classification divides HCA into five categories: HHCA, $\beta$-HCA, IHCA, ShHCA and unclassified HCA. HCA with inactivate biallelic mutations of hepatocyte nuclear factor $1 \alpha$ (HNF1A) are defined as HHCA. ${ }^{13-15} \mathrm{HCA}$ with mutations of $\beta$-catenin (CTNNB1) are referred to as $\beta$-HCA. Inflammatory HCA results from mutations that activate the IL-6/JAK/STAT pathway. ${ }^{16}$ HCA with activated sonic hedgehog pathway is called ShHCA. The remainder that lacks the above characteristics is defined as unclassified HCA. ${ }^{17} \beta$-HCA has two subtypes, according to their mutation loci. Mutations in exon 3 and exon $7 / 8$ are responsible for $b^{\mathrm{ex}^{3}} \mathrm{HCA}$ and $b^{\mathrm{ex} 7,8} \mathrm{HCA}$, respectively. $b^{e x^{3}} \mathrm{HCA}$ has a high risk of malignant transformation into HCC. However, $b^{\text {ex } 7,8} \mathrm{HCA}$ has no correlation with malignant transformation. Half of $b^{\text {ex }}{ }^{3} \mathrm{HCA}$ and $b^{\mathrm{ex} 7,8} \mathrm{HCA}$ pres-

Received: December 26, 2018; Revised: January 21, 2019; Accepted: January 30, 2019

Corresponding author: Hee-Jung Wang

Division of Hepatobiliary Surgery and Liver Transplantation, Department of Surgery, Ajou University School of Medicine, 164 Worldcup-ro, Yeongtong-gu, Suwon 16499, Korea

Tel: +82-31-219-5204, Fax: +82-31-219-5755, E-mail: wanghj@ajou.ac.kr

Copyright (C) 2019 by The Korean Association of Hepato-Biliary-Pancreatic Surgery

This is an Open Access article distributed under the terms of the Creative Commons Attribution Non-Commercial License (http://creativecommons.org/ licenses/by-nc/4.0) which permits unrestricted non-commercial use, distribution, and reproduction in any medium, provided the original work is properly cited. Annals of Hepato-Biliary-Pancreatic Surgery - pISSN: 2508-5778 - elSSN: 2508-5859 
ents with inflammation, which can be classified as $b^{\mathrm{ex}^{3}}$ IHCA and $b^{\text {ex7,8}}$ IHCA, respectively. ${ }^{16,18}$ We reviewed a series of nine patients who underwent liver surgery in our department due to the existence of clinical symptoms. The present study was designed to analyze the characteristics of HCA by performing molecular classification.

\section{MATERIALS AND METHODS}

\section{Patient characteristics}

We collected data from nine patients diagnosed with HCA by pathological examination in our department. Patients underwent liver resection between 1995 and 2016 due to the existence of clinical symptoms. We collected clinical data including age, sex, preoperative tests (creatine, albumin, total bilirubin, aspartate aminotransferase, alanine aminotransferase, $\alpha$-fetoprotein, prothrombin time, international normalized ratio, and indocyanine green retention after $15 \mathrm{~min}$ ), presence of hepatitis and GSD, mean tumor size, tumor number and recurrence, and disease-free and overall survival. This study was approved by the Institutional Review Board (IRB) of our institution (IRB number AJIRB-BMR-KSP-18-201).

Preoperative computed tomography and/or magnetic resonance imaging were performed to evaluate tumor number, size and location, and intraoperative ultrasonography was performed to double check the tumor characteristics more precisely. The surgical specimens were reviewed by pathological specialists. Diagnosis of HCA was confirmed according to typical tumor histological features. Malignant transformation of HCA into HCC was characterized by foci of HCC surrounded by background HCA cells. Immunohistochemistry was performed on the formalin-fixed, paraffin-embedded blocks to classify the categories of HCA, according to the 2010 World Health Organization guidelines. ${ }^{19}$

\section{Immunohistochemical (IHC) staining}

The following markers were used: $\beta$-catenin $(1: 100$, mouse monoclone 14; BD Biosciences, Franklin Lakes, NJ, USA); glutamine synthetase (GS) (1:300, mouse monoclone 6 ; BD Biosciences); liver fatty acid-binding protein (LFABP) (1:1000, mouse monoclone L2B10; Abcam, Cambridge, UK); serum amyloid-A (SAA) (prediluted, mouse monoclone mc1; Dako, Glostrup, Denmark); and
C-reactive protein (CRP) (1:1000, rabbit polyclonal; Abcam). Heat-mediated antigen retrieval with citrate buffer ( $\mathrm{pH} \mathrm{6)}$ before commencing IHC staining protocol was performed. Samples were incubated with primary antibody for $30 \mathrm{~min}$ at room temperature. In $\beta$-catenin activated mutation, nuclear staining of $\beta$-catenin or overexpression of GS was interpreted as positive. LFABP was considered positive when HCA and non-tumor hepatocytes expressed protein simultaneously. SAA was interpreted as positive if $>10 \%$ of adenomas were stained. CRP was used to complement SAA in diagnosis of IHCA.

In order to find out the correlation with malignant transformation, the patients were divided into malignant transformation and non-malignant transformation groups. Results of preoperative clinical test, follow-up data and history of exposure to exogenous steroids were recorded.

\section{Statistical analysis}

For continuous variables, data were synopsized as mean ( \pm standard deviation), and for categorical variables, data were processed by frequency and percentage. Statistical analysis was done using SPSS for Windows version 20.0 (SPSS, Chicago, IL, USA).

Table 1. Characteristics of 9 patients with hepatocellular adenoma

\begin{tabular}{lllr}
\hline \multicolumn{1}{c}{ Characteristics } & $\begin{array}{c}\text { Number } \\
(\%)\end{array}$ & $\begin{array}{c}\text { Mean } \\
( \pm \text { SD) }\end{array}$ & range \\
\hline Age (years) & & $43.4 \pm 16.8$ & $20-74$ \\
Sex & $7(77.8)$ & & \\
$\quad$ Male & $2(22.2)$ & & \\
$\quad$ Female & $0(0)$ & & \\
Hepatitis & $0(0)$ & & \\
Anabolic steroids & $0(0)$ & & \\
Oral contraceptives & $1(11.1)$ & & \\
Glycogen storage disease & $7(77.8)$ & & \\
Malignant transformation & & $9.8 \pm 4.1$ & $4.7-14.5$ \\
HCA size (cm) & & & \\
The number of HCA & $8(88.9)$ & & \\
$\quad$ Solitary HCA & $1(11.1)$ & & \\
$\quad$ Multiple HCA & $2(22.2)$ & & \\
Recurrence & & $90.5 \pm 55.9$ & $52-131$ \\
Recurrence after surgery (mo) & & $72.3 \pm 54.5$ & $9-172$ \\
Disease free survival (mo) & & $91.4 \pm 69.8$ & $9-189$ \\
Overall survival (mo) & &
\end{tabular}

Data is shown as number (\%) or mean $\pm \mathrm{SD}$ HCA, hepatocellular adenoma 


\section{RESULTS}

\section{Clinical data}

The characteristics of nine patients are summarized in Table 1. Patients' age ranged from 20 to 74 years; two of them were aged $>50$ years, a 57-year-old man and a 74-year-old woman. None of them had hepatitis B or C virus infection. Malignant transformation occurred in six male and one female patients. Two female patients had no history of OCs administration. One male patient was diagnosed with GSD before liver surgery. The mean size of HCA was $9.8 \mathrm{~cm}$ (range 4.7-14.5 cm). Eight patients had solitary HCA and one patient had two HCAs. Recurrence occurred in two patients at 52 and 131 months after liver surgery, respectively. The mean disease-free survival of all patients was 72.3 months (range 9-172 months) and overall survival was 91.4 months (range 9-189 months).

To evaluate the differences and features of malignant transformation of HCA into HCC, nine patients were div- ided into malignant transformation and non-malignant transformation groups (Table 2). The mean age was 48.3 $\mathrm{y}$ in malignant transformation group, and $26.5 \mathrm{y}$ in non-malignant transformation group. The mean size of HCA was $11.2 \mathrm{~cm}$ in malignant group and $4.9 \mathrm{~cm}$ in non-malignant group, respectively. Male to female ratio was 6:1 in the malignant transformation group. GSD was found in one patient in the malignant transformation group. Malignant transformation developed in six patients with solitary HCA and one patient with two HCAs. Recurrence was found in one patient in the malignant transformation group at 52 months and in one patient in the non-malignant transformation group at 131 months after liver surgery.

\section{Pathological data}

IHC staining was performed using antibodies with $\beta$-catenin, SAA, CRP, GS, LFABP and to classify the categories of HCA (Fig. 1). All nine patients were negative for $\beta$-catenin antibody staining. Further, to exam the cat-

Table 2. Characteristics of malignant transformation group and non-malignant transformation group

\begin{tabular}{lcc}
\hline \multicolumn{1}{c}{ Characteristics } & Malignant $(\mathrm{n}=7)$ & Non-malignant $(\mathrm{n}=2)$ \\
\hline Age (years), mean $\pm \mathrm{SD}$ & $48.3 \pm 15.5$ & $26.5 \pm 9.2$ \\
Sex & & $1(50)$ \\
Male, n (\%) & $6(85.7)$ & $1(50)$ \\
Female, n (\%) & $1(14.3)$ & 0.8 \\
Preoperative tests & & $4.7 \pm 0.2$ \\
Creatine (mg/dl), mean $\pm \mathrm{SD}$ & $0.8 \pm 0.2$ & $1.3 \pm 1.1$ \\
Albumin (g/d), mean $\pm \mathrm{SD}$ & $3.5 \pm 1.0$ & $25.5 \pm 12.0$ \\
Total bilirubin (mg/dl), mean $\pm \mathrm{SD}$ & $0.8 \pm 0.4$ & $49.0 \pm 36.8$ \\
AST (U/L), mean $\pm \mathrm{SD}$ & $144.2 \pm 153.0$ & $1.5 \pm 0.1$ \\
ALT (U/L), mean $\pm \mathrm{SD}$ & $87.8 \pm 83.1$ & $12.6 \pm 0.6$ \\
AFP (ng/mL), mean $\pm \mathrm{SD}$ & $2.4 \pm 1.6$ & $1.1 \pm 0.1$ \\
PT (sec), mean $\pm \mathrm{SD}$ & $11.5 \pm 1.0$ & $7.0 \pm 3.7$ \\
INR, mean $\pm \mathrm{SD}$ & $1.0 \pm 0.1$ & $0(0)$ \\
ICG R15 (\%), mean $\pm \mathrm{SD}$ & $6.7 \pm 3.8$ & $4.9 \pm 0.2$ \\
Glycogen storage disease & $1(14.3)$ & $2(100)$ \\
Mean HCA size (cm), mean $\pm \mathrm{SD}$ & $11.2 \pm 3.5$ & $0(0)$ \\
The number of HCA & & $1(50)$ \\
Solitary HCA & $6(85.7)$ & 131 \\
Multiple HCA & $1(14.3)$ & $116.0 \pm 21.2$ \\
Recurrence & $1(14.3)$ & $133.5 \pm 46.0$ \\
Recurrence after surgery (mo) & 52 & \\
Disease free survival (mo), mean $\pm \mathrm{SD}$ & $59.9 \pm 55.4$ & \\
Overall survival (mo), mean $\pm \mathrm{SD}$ & $79.4 \pm 73.4$ & \\
\hline
\end{tabular}

Data is shown as number (\%) or mean $\pm \mathrm{SD}$

ALT, alanine aminotransferase; AST, aspartate aminotransferase; AFP, alpha-fetoprotein; PT, prothrombin time; INR, international normalized ratio; ICG R15, indocyanine green retention rate at $15 \mathrm{~min}$ 

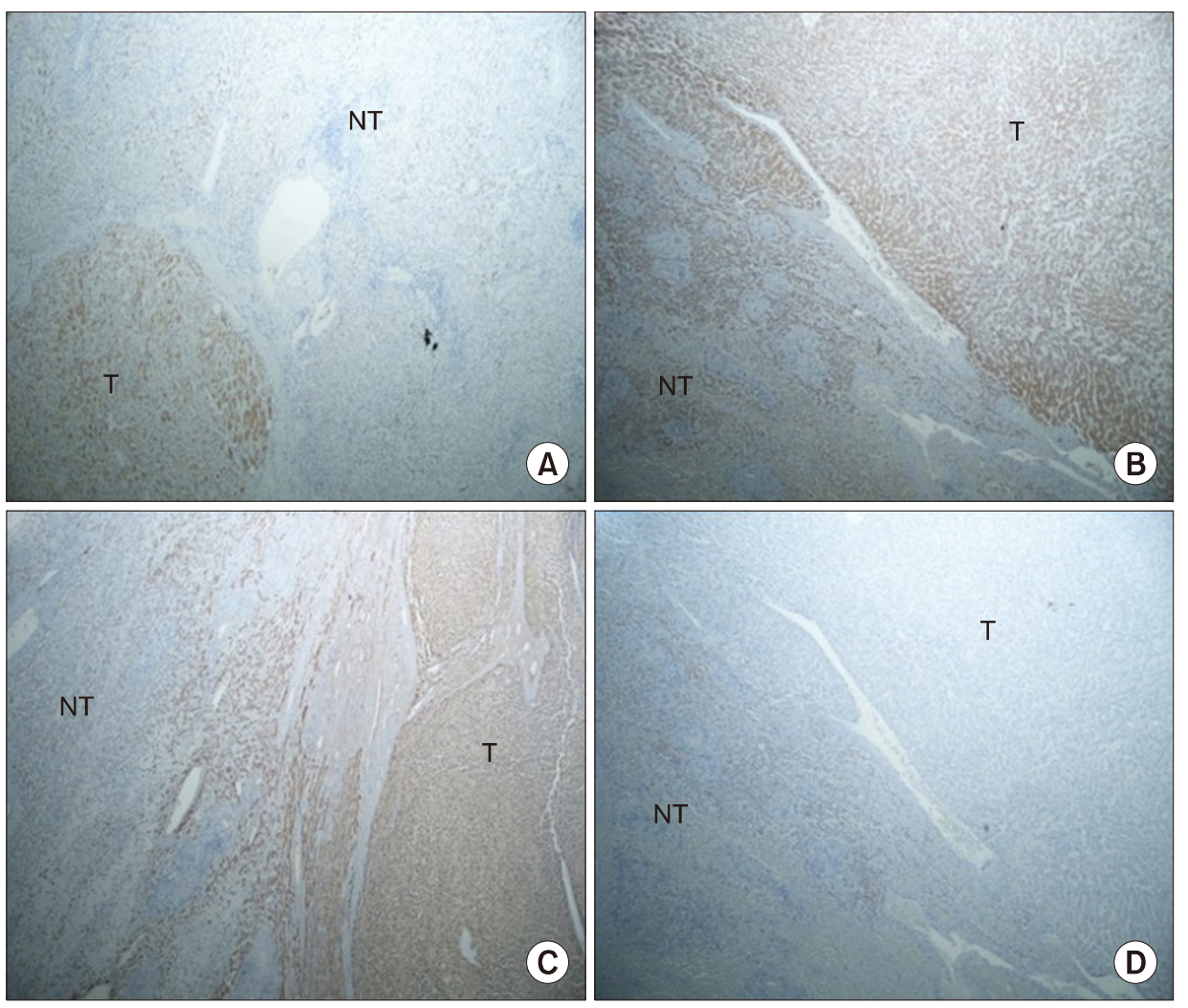

Fig. 1. The results of immunohistochemical staining. (A) Serum amyloid-A (100×): HCA shows diffuse expression compared with non-tumor liver tissue. (B) Creactive protein $(100 \times)$ : strong overexpression is observed in HCA compared with non-tumor liver tissue. (C) Glutamine synthetase $(100 \times)$ : HCA shows diffuse overexpression in contrast to the non-tumor liver tissue. (D) Liver fatty acid-binding protein (LFABP) $(100 \times)$ : loss of LFABP expression in HCA is shown compared with nontumor liver tissue.

Table 3. Result of immunohistochemical staining

\begin{tabular}{cccccc}
\hline Patients & $\begin{array}{c}\text { Glutamine } \\
\text { synthetase }\end{array}$ & $\beta$-catenin & $\begin{array}{c}\text { C-reactive } \\
\text { protein }\end{array}$ & $\begin{array}{c}\text { Serum } \\
\text { amyloid-A }\end{array}$ & $\begin{array}{c}\text { Liver-specific fatty } \\
\text { acid binding protein }\end{array}$ \\
\hline A & + & - & + & + & + \\
B & + & - & + & + & + \\
C & + & - & - & + & + \\
D & + & - & + & + & + \\
E & - & - & + & + & + \\
F & + & - & + & + & + \\
G & + & - & + & + & - \\
H & - & - & + & + & + \\
I & - & & & + & + \\
\hline
\end{tabular}

enin $\beta 1$ mutation, we used GS staining for the diagnosis. Results of staining are displayed in Table 3. Four patients with both $\beta$-catenin and inflammation were classified as $\beta$-IHCA. Two patients were defined as $\beta$-HCA. Two patients were classified as HHCA. Only one patient was defined as IHCA. None of the patients had unclassified HCA. From the perspective of malignant transformation, $\beta$-catenin activated mutation was detected in six of seven patients, one patient with underlying GSD was classified as IHCA, that had a malignant transformation into HCC.

\section{DISCUSSION}

HCA is a rare benign tumor and malignant transformation into HCC is even rarer. The first reported case of malignant transformation of HCA into HCC was in a 21-year old woman with a history of using OCs for two years. ${ }^{20}$ From a systematic review of the past 40 years, malignant transformation of HCA into HCC occurred only in 68 of $1635 \mathrm{HCA}(4.2 \%){ }^{21}$ In recent studies, six of 128 , ten of 122, five of 124, and 23 of 218 patients underwent malignant transformation of $\mathrm{HCA}$ into $\mathrm{HCC}$, with an in- 
cidence of $4.7-10.6 \%$. In our study, malignant transformation occurred in seven of nine patients. Notably, in contrast to the high percentage of malignant transformation in HCA, the actual number of malignant transformations was similar to that in the previous large cohort study. It can be interpreted as the low incidence of HCA in women from Eastern countries, whom accounting for approximately $90 \%$ of the proportion of HCA. Because of the differences in culture and healthcare systems, OCs use is lower in Eastern countries, leading to a high frequency of HCA in Western compared with Eastern countries.

Based on previous studies, male sex, large tumor size, exposure to steroids, underlying metabolic syndrome and activated $\beta$-catenin mutation are risk factors for malignant transformation of HCA into HCC. ${ }^{22}$ Before 2000, several studies addressed malignant transformation of HCA in women. However, the incidence of malignant transformation in men has increased and exceeded that of women since 2000. The relative risk of malignant transformation in men was ten times higher than in women. An equivalent result was obtained in our study: one male patient underwent malignant transformation in 1995, and the remaining six male patients developed malignant transformation after 2000. Six of seven male patients presented with malignant transformation. Although it was not significant because of the small number of patients, we considered male sex to be a risk factor for malignant transformation of HCA into HCC.

There have been a few studies on the correlation between tumor size of HCA and the risk of malignant transformation. In a systematic review of HCA including 68 patients with malignant transformation into HCC, only three patients $(4.4 \%)$ had a tumor size $<5 \mathrm{~cm}^{20}$ In our series, although there is no statistical basis due to the small number of cases, we found that the mean tumor size was $11.2 \mathrm{~cm}$ in the malignant transformation group, greater than non-malignant transformation group.

Metabolic syndrome was considered to be a new risk factor for malignant transformation. Underlying GSD is a known strong risk factor for development of HCA; however, malignant transformation was not observed during follow-up. ${ }^{23}$ There are a few reports of malignant transformation of HCA into $\mathrm{HCC}$ in patients with GSD. ${ }^{24-26}$ In a study of 25 HCAs from 15 patients with GSD, HCAs were classified as $\beta$-HCA, IHCA and unclassified HCA by IHC staining, and no HNF1A inactivation was found. ${ }^{27}$ In the present study, one patient with underlying GSD developed malignant transformation, and the HCA was classified as IHCA by IHC staining.

Catenin $\beta 1$ mutation is considered to be one of the main risk factors for malignant transformation of HCA into $\mathrm{HCC}^{28}$ Because of the low sensitivity of $\beta$-catenin staining in detecting mutation of catenin $\beta 1$, GS staining was used to assist the diagnosis. Among seven patients in the malignant transformation group, six were associated with mutation of $\beta$-catenin, including four with $\beta$-IHCA and two with $\beta$-HCA. Surprisingly, in respect of detecting catenin $\beta 1$ mutation, all six HCAs were negative for $\beta$-catenin staining but positive for GS staining. We consider that GS staining appeared to be more sensitive than $\beta$-catenin staining in detecting catenin $\beta 1$ mutation.

IHCA accounts for $35 \%$ of all HCA. Half of the $b^{e x^{3}} \mathrm{HCA}$ and $b^{\mathrm{ex} 7,8} \mathrm{HCA}$ present with the inflammatory phenotype simultaneously, and the subgroups of IHCA can be defined as $b^{e^{3}}$ IHCA and $b^{\text {ex7,8 }}$ IHCA, respectively. Malignant transformation has not been found in the subgroup of IHCA that is negative for catenin $\beta 1$ mutation. However, we found that the patient $\mathrm{E}$ in Table 3 was classified as IHCA with absence of catenin $\beta 1$ mutation (negative for $\beta$-catenin and GS IHC staining), that had a malignant transformation. We consider that there is an unknown pathway regulating malignant transformation in IHCA.

Our study revealed that the mean tumor size and activation of catenin $\beta 1$ mutation status might be the risk factors for the malignant transformation of HCA into HCC. Interestingly, a single patient with underlying GSD was classified as IHCA, that had a malignant transformation to HCC. Our results support further studies of the correlation between IHCA and HCC.

\section{REFERENCES}

1. Rooks JB, Ory HW, Ishak KG, Strauss LT, Greenspan JR, Hill AP, et al. Epidemiology of hepatocellular adenoma. The role of oral contraceptive use. JAMA 1979;242:644-648.

2. Heinemann LA, Weimann A, Gerken G, Thiel C, Schlaud M, DoMinh T. Modern oral contraceptive use and benign liver tumors: the German Benign Liver Tumor Case-Control Study. Eur J Contracept Reprod Health Care 1998;3:194-200.

3. Sasaki M, Nakanuma Y. Overview of hepatocellular adenoma in 
Japan. Int J Hepatol 2012;2012:648131.

4. Matsumoto Y, Yamabe S, Sugishima T, Geronazzo D. Perception of oral contraceptives among women of reproductive age in Japan: a comparison with the USA and France. J Obstet Gynaecol Res 2011;37:887-892.

5. Svrcek M, Jeannot E, Arrivé L, Poupon R, Fromont G, Fléjou $\mathrm{JF}$, et al. Regressive liver adenomatosis following androgenic progestin therapy withdrawal: a case report with a 10-year follow-up and a molecular analysis. Eur J Endocrinol 2007; 156:617-621.

6. Reddy SK, Kishnani PS, Sullivan JA, Koeberl DD, Desai DM, Skinner MA, et al. Resection of hepatocellular adenoma in patients with glycogen storage disease type Ia. J Hepatol 2007;47: 658-663.

7. Ozenne V, Paradis V, Vullierme MP, Vilgrain V, Leblanc T, Belghiti J, et al. Liver tumours in patients with Fanconi anaemia: a report of three cases. Eur J Gastroenterol Hepatol 2008;20: 1036-1039.

8. Schmidt J. Systematic review of haemorrhage and rupture of hepatocellular adenomas. Br J Surg 2012;99:911-916.

9. Bioulac-Sage P, Laumonier H, Couchy G, Le Bail B, Sa Cunha A, Rullier A, et al. Hepatocellular adenoma management and phenotypic classification: the Bordeaux experience. Hepatology 2009;50:481-489.

10. Dokmak S, Paradis V, Vilgrain V, Sauvanet A, Farges O, Valla $\mathrm{D}$, et al. A single-center surgical experience of 122 patients with single and multiple hepatocellular adenomas. Gastroenterology 2009; 137:1698-1705.

11. Deneve JL, Pawlik TM, Cunningham S, Clary B, Reddy S, Scoggins CR, et al. Liver cell adenoma: a multicenter analysis of risk factors for rupture and malignancy. Ann Surg Oncol 2009; 16:640-648.

12. Farges O, Ferreira N, Dokmak S, Belghiti J, Bedossa P, Paradis V. Changing trends in malignant transformation of hepatocellular adenoma. Gut 2011;60:85-89.

13. Bacq Y, Jacquemin E, Balabaud C, Jeannot E, Scotto B, Branchereau $\mathrm{S}$, et al. Familial liver adenomatosis associated with hepatocyte nuclear factor 1alpha inactivation. Gastroenterology 2003;125: 1470-1475.

14. Bluteau O, Jeannot E, Bioulac-Sage P, Marqués JM, Blanc JF, Bui $\mathrm{H}$, et al. Bi-allelic inactivation of TCF1 in hepatic adenomas. Nat Genet 2002;32:312-315.

15. Jeannot E, Mellottee L, Bioulac-Sage P, Balabaud C, Scoazec JY, Tran Van Nhieu J, et al. Spectrum of HNF1A somatic mutations in hepatocellular adenoma differs from that in patients with MODY3 and suggests genotoxic damage. Diabetes 2010;59: 1836-1844.

16. Zucman-Rossi J, Jeannot E, Nhieu JT, Scoazec JY, Guettier C, Rebouissou S, et al. Genotype-phenotype correlation in hep- atocellular adenoma: new classification and relationship with HCC. Hepatology 2006;43:515-524.

17. Nault JC, Couchy G, Balabaud C, Morcrette G, Caruso S, Blanc $\mathrm{JF}$, et al. Molecular classification of hepatocellular adenoma associates with risk factors, bleeding, and malignant transformation. Gastroenterology 2017;152:880-894.

18. Pilati C, Letouzé E, Nault JC, Imbeaud S, Boulai A, Calderaro $\mathrm{J}$, et al. Genomic profiling of hepatocellular adenomas reveals recurrent FRK-activating mutations and the mechanisms of malignant transformation. Cancer Cell 2014;25:428-441.

19. Shafizadeh N, Genrich G, Ferrell L, Kakar S. Hepatocellular adenomas in a large community population, 2000 to 2010: reclassification per current World Health Organization classification and results of long-term follow-up. Hum Pathol 2014;45: 976-983.

20. Davis M, Portmann B, Searle M, Wright R, Williams R. Histological evidence of carcinoma in a hepatic tumour associated with oral contraceptives. Br Med J 1975;4:496-498.

21. Stoot JH, Coelen RJ, De Jong MC, Dejong CH. Malignant transformation of hepatocellular adenomas into hepatocellular carcinomas: a systematic review including more than 1600 adenoma cases. HPB (Oxford) 2010;12:509-522.

22. Gorayski P, Thompson CH, Subhash HS, Thomas AC. Hepatocellular carcinoma associated with recreational anabolic steroid use. Br J Sports Med 2008;42:74-75; discussion 75.

23. Labrune $\mathrm{P}$, Trioche $\mathrm{P}$, Duvaltier I, Chevalier $\mathrm{P}$, Odièvre $\mathrm{M}$. Hepatocellular adenomas in glycogen storage disease type I and III: a series of 43 patients and review of the literature. J Pediatr Gastroenterol Nutr 1997;24:276-279.

24. Franco LM, Krishnamurthy V, Bali D, Weinstein DA, Arn P, Clary B, et al. Hepatocellular carcinoma in glycogen storage disease type Ia: a case series. J Inherit Metab Dis 2005;28:153-162.

25. Baheti AD, Yeh MM, O'Malley R, Lalwani N. Malignant transformation of hepatic adenoma in glycogen storage disease type-1a: report of an exceptional case diagnosed on surveillance imaging. J Clin Imaging Sci 2015;5:47.

26. Cassiman D, Libbrecht L, Verslype C, Meersseman W, Troisi $\mathrm{R}$, Zucman-Rossi J, et al. An adult male patient with multiple adenomas and a hepatocellular carcinoma: mild glycogen storage disease type Ia. J Hepatol 2010;53:213-217.

27. Calderaro J, Labrune P, Morcrette G, Rebouissou S, Franco D, Prévot S, et al. Molecular characterization of hepatocellular adenomas developed in patients with glycogen storage disease type I. J Hepatol 2013;58:350-357.

28. Bioulac-Sage P, Rebouissou S, Thomas C, Blanc JF, Saric J, Sa Cunha A, et al. Hepatocellular adenoma subtype classification using molecular markers and immunohistochemistry. Hepatology 2007;46:740-748. 